\title{
Sivers effect in semi-inclusive deeply inelastic scattering
}

\author{
J. C. Collins, ${ }^{1,3}$ A. V. Efremov, ${ }^{2}$ K. Goeke, ${ }^{3}$ S. Menzel,${ }^{3}$ A. Metz,${ }^{3}$ and P. Schweitzer ${ }^{3}$ \\ ${ }^{1}$ Penn State University, 104 Davey Lab, University Park PA 16802, U.S.A. \\ ${ }^{2}$ Joint Institute for Nuclear Research, Dubna, 141980 Russia \\ ${ }^{3}$ Institut für Theoretische Physik II, Ruhr-Universität Bochum, D-44780 Bochum, Germany
}

(Dated: November 2005)

\begin{abstract}
The Sivers function is extracted from HERMES data on single spin asymmetries in semi-inclusive deeply inelastic scattering. Our analysis use a simple Gaussian model for the distribution of transverse parton momenta, together with the flavor dependence given by the leading $1 / N_{c}$ approximation and a neglect of the Sivers antiquark distribution. We find that within the errors of the data these approximations are sufficient.
\end{abstract}

PACS numbers: 13.88.+e, 13.85.Ni, 13.60.-r, 13.85.Qk

\section{INTRODUCTION}

Single spin asymmetries (SSA) in hard reactions have a long history dating back to the 1970s when significant polarizations of $\Lambda$-hyperons in collisions of unpolarized hadrons were observed [1], and to the early 1990s when large asymmetries in $p^{\uparrow} p \rightarrow \pi X$ or $p^{\uparrow} \bar{p} \rightarrow \pi X$ were found at FNAL [2]. No fully consistent and satisfactory unifying approach to the theoretical description of these observations has been found so far — see the reviews [3].

Interestingly, the most recently observed SSA phenomena, namely those in semi-inclusive deeply inelastic scattering (SIDIS) [4-11], seem better under control. This is in particular the case for the transverse target SSA observed at HERMES and COMPASS [8-11]. On the basis of a generalized factorization approach in which transverse parton momenta are taken into account [12-14] these "leading twist" asymmetries can be explained [15] in terms of the Sivers [16-19] or Collins effect [20]. The former describes, loosely speaking, the distribution of unpolarized partons in a transversely polarized proton, the latter describes the fragmentation of transversely polarized partons into unpolarized hadrons. In the transverse target SSA these effects can be distinguished by the different azimuthal angle distribution of the produced hadrons: Sivers effect $\propto \sin \left(\phi-\phi_{S}\right)$, while Collins effect $\propto \sin \left(\phi+\phi_{S}\right)$, where $\phi$ and $\phi_{S}$ denote respectively the azimuthal angles of the produced hadron and the target polarization vector with respect to the axis defined by the hard virtual photon [15]. Both effects have been subject to intensive phenomenological studies in hadron-hadron-collisions [21-25] and in SIDIS [26-35]. For the longitudinal target SSA in SIDIS, which were observed first [4-6] but are dominated by subleading-twist effects [36], the situation is less clear and their description (presuming the factorization theorems [12-14] can be generalized to twist-3) is more involved [37, 38].

In this work we will concentrate on the Sivers effect, which is quantified by the "Sivers function" $f_{1 T}^{\perp a}\left(x, \mathbf{p}_{T}^{2}\right)$ (in the notation recommended in [39]). It is referred to as "naively" or "artificially time-reversal-odd" for it arises from a correlation between the nucleon spin $\mathbf{S}_{\mathrm{T}}$ and the intrinsic transverse parton momentum $\mathbf{p}_{\mathrm{T}}$, both transverse with respect to the nucleon momentum $\mathbf{P}_{N}$ in the infinite momentum frame, with the effect being proportional to $\left(\mathbf{S}_{\mathrm{T}} \times \mathbf{p}_{\mathrm{T}}\right) \cdot \mathbf{P}_{N}$.

By adequately weighting the events entering the spin asymmetry with $\sin \left(\phi-\phi_{S}\right)$ one can project out from the data the Sivers SSA. Including into the weight in addition to that a power of the transverse momentum of the produced hadron $P_{h \perp} \equiv\left|\mathbf{P}_{h \perp}\right|$ yields an SSA which is described (with the neglect of soft factors [12-14]) model-independently in terms of the "transverse moment" $f_{1 T}^{\perp(1) a}(x)$ of the Sivers function [15]. Preliminary HERMES data analyzed in this way were presented [8] and subject to first studies [32]. However, the currently available final HERMES and COMPASS data $[9,10]$ were analyzed without a transverse momentum weight, and can only be interpreted by resorting to some model for the distribution of the transverse parton momenta in the "unintegrated" [40] Sivers distribution and unpolarized fragmentation function. Different models have been explored in literature [33-35]. Here we approximate the distribution of transverse parton momenta in the Sivers function to be Gaussian.

We pay particular attention to the demonstration of the phenomenological consistency of the approach, and fix or constrain the free parameters in the Gaussian ansatz consistently by the SIDIS HERMES data. Although hereby the Gaussian width of the Sivers function remains poorly constrained, this does not prevent a meaningful extraction of the transverse moment of the Sivers distribution function from the data [9]. This demonstrates that — within the accuracy of the present data - the Gaussian ansatz is robust and reliable. A comparison to extractions of the Sivers function, where no [32] or other models [33-35] were assumed, helps to estimate the effects of model-dependence. We find them smaller than the statistical accuracy of the present data.

In order to reduce the number of free parameters in the ansatz for the Sivers function, we impose as an additional theoretical constraint the predictions for the Sivers function from the QCD limit of a large number of colours $N_{c}$ [41], 


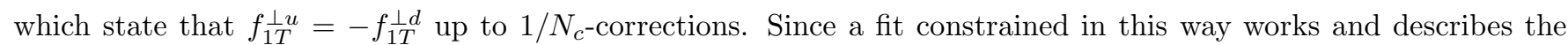
HERMES data [9], our study, as a byproduct, not only tests the large- $N_{c}$ results [41] but also naturally explains the smallness of the Sivers effect from a deuteron target observed by COMPASS [10]. Besides choosing different models for transverse parton momenta and/or ways to fix the respective parameters, the explicit use of the large- $N_{c}$ constraints is the main difference of our approach compared to the works [33-35].

For the fit we use only the HERMES data [9] on the $x$-dependence of the Sivers SSA. Thus, the HERMES data on the $z$-dependence serve as cross and consistency checks for the fit and the Gaussian ansatz. We also explicitly address the questions what could be the effects of $1 / N_{c}$-corrections and Sivers antiquarks (which we neglect in our fit). Finally, we suggest how the Gaussian ansatz could be further tested by means of SIDIS data. Such tests are of importance for they allow to understand the range of applicability and the limitations of this ansatz.

\section{SIVERS EFFECT IN SIDIS}

Consider the process $l p^{\uparrow} \rightarrow l^{\prime} h X$, where "^" denotes the transverse (with respect to the beam) target polarization. Let $P, l\left(l^{\prime}\right)$ and $P_{h}$ denote respectively the momentum of the target proton, incoming (outgoing) lepton and produced hadron. The relevant kinematic variables are $q=l-l^{\prime}$ with $Q^{2}=-q^{2}, x=Q^{2} /(2 P \cdot q)$ and $z=P \cdot P_{h} / P \cdot q$. The Sivers SSA as presented in Ref. [9] is defined as sum over events $i$ as follows:

$$
A_{U T}^{\sin \left(\phi-\phi_{S}\right)}=\frac{\sum_{i} \sin \left(\phi_{i}-\phi_{S, i}\right)\left[N^{\uparrow}\left(\phi_{i} ; \phi_{S, i}\right)-N^{\downarrow}\left(\phi_{i} ; \phi_{S, i}+\pi\right)\right]}{\frac{1}{2} \sum_{i}\left[N^{\uparrow}\left(\phi_{i} ; \phi_{S, i}\right)+N^{\downarrow}\left(\phi_{i} ; \phi_{S, i}+\pi\right)\right]},
$$

where $N^{\uparrow(\downarrow)}\left(\phi_{i} ; \phi_{S, i}\right)$ are the event counts for the respective target polarization (corrected for depolarization effects) - see Fig. 1 for the definition of kinematics. It is understood that if the SSA is considered as function of one kinematic variable, then an appropriate averaging over the other variables is implied.

In order to describe the Sivers SSA as defined in (1) we will make two major simplifications. The first consists in neglecting the soft factors [12-14], and their associated energy dependence. This step simplifies the description of the process considerably, though it is difficult to quantify the uncertainty we introduce in this way. Then, to leading order in the hard scale, the SSA is given by

$$
\begin{aligned}
& A_{U T}^{\sin \left(\phi_{h}-\phi_{S}\right)}=(-2) \times \\
& \frac{\sum_{a} e_{a}^{2} \int \mathrm{d}^{2} \mathbf{P}_{h \perp} \int \mathrm{d}^{2} \mathbf{p}_{T} \int \mathrm{d}^{2} \mathbf{K}_{T} \sin \left(\phi_{h}-\phi_{S}\right) \sin \left(\phi_{\mathbf{p}_{T}}-\phi_{S}\right) \frac{\left|\mathbf{p}_{T}\right|}{M_{N}} \delta^{(2)}\left(z \mathbf{p}_{T}+\mathbf{K}_{T}-\mathbf{P}_{h \perp}\right) x f_{1 T}^{\perp a}\left(x, \mathbf{p}_{T}^{2}\right) D_{1}^{a}\left(z, \mathbf{K}_{T}^{2}\right)}{\sum_{a} e_{a}^{2} x f_{1}^{a}(x) D_{1}^{a}(z)},
\end{aligned}
$$

where we cancelled out the $Q^{2}$ and $y$-dependent factors (which describe the unpolarized partonic subprocess and are the same in the numerator and denominator), and $\phi_{\mathbf{p}_{T}}$ denotes the azimuthal angle around the $z$-axis $\mathbf{e}_{3}$ of the parton struck from the target nucleon. In a full description of the process the transverse parton momenta in $f_{1 T}^{\perp a}\left(x, \mathbf{p}_{T}^{2}\right)$ and $D_{1}^{a}\left(z, \mathbf{K}_{T}^{2}\right)$ would be convoluted with the soft factors [12-14] instead of the simplifying $\delta$-function.

However, this simplification is not yet sufficient for the purpose of extracting the Sivers function. In the numerator on the right-hand-side of Eq. (2) the integrals convoluting the transverse momenta cannot be solved, unless one knows $f_{1 T}^{\perp a}\left(x, \mathbf{p}_{T}^{2}\right)$ and $D_{1}^{a}\left(z, \mathbf{K}_{T}^{2}\right)$, which is not the case. The situation would be different if in the SSA in Eq. (1) in addition had been introduced a power of the transverse hadron momentum $\left|\mathbf{P}_{h \perp}\right|$.

The leading order expression for such a weighted SSA is given by [15]

$$
A_{U T, \pi}^{\sin \left(\phi-\phi_{S}\right) \frac{P_{h} \perp}{M_{N}}}(x)=(-2) \frac{\sum_{a} e_{a}^{2} x f_{1 T}^{\perp(1) a}(x) z D_{1}^{a / \pi}(z)}{\sum_{a} e_{a}^{2} x f_{1}^{a}(x) D_{1}^{a / \pi}(z)},
$$

where the transverse moment of the Sivers function is defined as

$$
f_{1 T}^{\perp(1) a}(x) \equiv \int \mathrm{d}^{2} \mathbf{p}_{T} \frac{\mathbf{p}_{T}^{2}}{2 M_{N}^{2}} f_{1 T}^{\perp a}\left(x, \mathbf{p}_{T}^{2}\right) .
$$

Preliminary HERMES data on the SSA weighted in this way were presented in [8]. From the point of view of minimizing the model-dependence in the analysis [32] it is preferable to consider data on an appropriately transverse-momentum-weighted SSA [15], such as in Eq. (3). However, a wealth of data on the SSA in SIDIS have been analyzed and presented without the "appropriate transverse momentum weights" [4-7, 9-11].

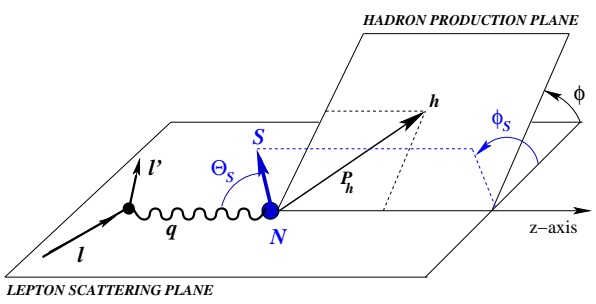

FIG. 1: Kinematics of the SIDIS process $l p \rightarrow l^{\prime} h X$ and the definitions of the azimuthal angles in the lab frame. 
Many models for the transverse momentum dependence of distribution and fragmentation functions were considered in literature [26-31, 33-35]. Among the most popular models is the Gaussian ansatz, which has two important virtues. It describes successfully the distributions of low (with respect to the relevant hard scale $Q$ ) transverse momenta in various hard reactions - see, for example, Ref. [23]. It also allows us to perform analytically the integrals over transverse momentum. In the Gaussian ansatz one assumes that the transverse momentum and $x$ - or $z$-dependence of distribution or fragmentation functions factorize, and that the distributions of the transverse parton momenta are Gaussian:

$$
\begin{aligned}
f_{1}^{a}\left(x, \mathbf{p}_{T}^{2}\right) & \equiv f_{1}^{a}(x) \frac{\exp \left(-\mathbf{p}_{T}^{2} / p_{\mathrm{unp}}^{2}\right)}{\pi p_{\mathrm{unp}}^{2}} \\
f_{1 T}^{\perp a}\left(x, \mathbf{p}_{T}^{2}\right) & \equiv f_{1 T}^{\perp a}(x) \frac{\exp \left(-\mathbf{p}_{T}^{2} / p_{\text {Siv }}^{2}\right)}{\pi p_{\text {Siv }}^{2}} \\
D_{1}^{a}\left(z, \mathbf{K}_{T}^{2}\right) & \equiv D_{1}^{a}(z) \frac{\exp \left(-\mathbf{K}_{T}^{2} / K_{D_{1}}^{2}\right)}{\pi K_{D_{1}}^{2}}
\end{aligned}
$$

The Gaussian widths $p_{\text {unp }}^{2}$ and $K_{D_{1}}^{2}$ are also referred to as mean square transverse momenta of the unpolarized distribution and fragmentation functions, respectively, since for example

$$
\left\langle p_{T}^{2}\right\rangle_{\text {unp }} \equiv \frac{\int \mathrm{d}^{2} \mathbf{p}_{T} \mathbf{p}_{T}^{2} f_{1}^{a}\left(x, \mathbf{p}_{T}^{2}\right)}{\int \mathrm{d}^{2} \mathbf{p}_{T} f_{1}^{a}\left(x, \mathbf{p}_{T}^{2}\right)} \stackrel{\text { Gauss }}{=} p_{\text {unp }}^{2}
$$

In general, the mean square transverse momenta could be flavour and $x$ - or $z$-dependent, a possibility that we will disregard. For later convenience let us also introduce the notion of a mean parton transverse momentum generally defined, and in the Gaussian model given as follows:

$$
\left\langle p_{T}\right\rangle_{\text {unp }} \equiv \frac{\int \mathrm{d}^{2} \mathbf{p}_{T}\left|\mathbf{p}_{T}\right| f_{1}^{a}\left(x, \mathbf{p}_{T}^{2}\right)}{\int \mathrm{d}^{2} \mathbf{p}_{T} f_{1}^{a}\left(x, \mathbf{p}_{T}^{2}\right)} \stackrel{\text { Gauss }}{=} \frac{\sqrt{\pi}}{2} p_{\text {unp }} .
$$

Under the above assumptions the expression for the Sivers SSA in Eqs. (1, 2) is given by [29]

$$
A_{U T}^{\sin \left(\phi-\phi_{S}\right)}=(-2) \frac{a_{\text {Gauss }} \sum_{a} e_{a}^{2} x f_{1 T}^{\perp(1) a}(x) D_{1}^{a}(z)}{\sum_{a} e_{a}^{2} x f_{1}^{a}(x) D_{1}^{a}(z)}, \quad \text { with } \quad a_{\text {Gauss }}=\frac{\sqrt{\pi}}{2} \frac{M_{N}}{\sqrt{p_{\text {Siv }}^{2}+K_{D_{1}}^{2} / z^{2}}} .
$$

Here we ignore the resolution cuts applied by the experiments: $P_{h \perp} \gtrsim 50 \mathrm{MeV}$ at HERMES [5, 9], and $P_{h \perp}>100 \mathrm{MeV}$ at COMPASS [10]. Taking such cuts into account would yield the same expression (8), however, with an $a_{\text {Gauss }}$ given in terms of incomplete $\Gamma$-functions. The error introduced here by neglecting these cuts, however, is marginal as we shall estimate below. In the Gaussian ansatz (5) the transverse moment of the Sivers function (4) is given by

$$
f_{1 T}^{\perp(1) a}(x) \stackrel{\text { Gauss }}{=} \frac{p_{\text {Siv }}^{2}}{2 M_{N}^{2}} f_{1 T}^{\perp a}(x) .
$$

The reason why in Eq. (8) we prefer to work with $f_{1 T}^{\perp(1) a}(x)$ instead of $f_{1 T}^{\perp a}(x)$ will become clear later. Before we start to extract the Sivers function from the HERMES data [9], it is necessary to fix or constrain the free parameters $p_{\text {Siv }}^{2}$ and $K_{D_{1}}^{2}$, preferably by (other) HERMES data for sake of consistency. The next Section is devoted to this task.

\section{UNPOLARIZED SIDIS, POSITIVITY CONSTRAINTS, AND THE LARGE $N_{c}$-LIMIT}

Let us assume the distribution of transverse momenta in $f_{1}^{a}\left(x, \mathbf{p}_{T}^{2}\right)$ and $D_{1}^{a}\left(z, \mathbf{K}_{T}^{2}\right)$ to be Gaussian according to (5), and let us furthermore assume the corresponding Gaussian widths to be flavour and $x$ - or $z$-independent. Then the average transverse momentum of the produced hadrons as function of $z$ is given by

$$
\left\langle P_{h \perp}(z)\right\rangle=\frac{\sqrt{\pi}}{2} \sqrt{z^{2} p_{\mathrm{unp}}^{2}+K_{D_{1}}^{2}} .
$$

Fig. 2 shows the HERMES data on $\left\langle P_{h \perp}(z)\right\rangle$ for $h=$ pions from Ref. [6]. Strictly speaking these data were taken from a deuterium target, but we will ignore this fact (i.e., we neglect nuclear binding effects and use isospin symmetry). 
Also we will ignore the fact that these mean values are not corrected for acceptance effects. What is important for us is that these data allow to fix the free parameters $p_{\text {unp }}^{2}$ and $K_{D_{1}}^{2}$. A best fit yields

$$
\begin{aligned}
p_{\text {unp }}^{2} & =0.33 \mathrm{GeV}^{2}, \\
K_{D_{1}}^{2} & =0.16 \mathrm{GeV}^{2},
\end{aligned}
$$

and is shown in Fig. (2) by a dashed line. We observe a good and for our purposes sufficient agreement. It is important to stress that the agreement could be improved at the prize of introducing a $z$-and/or flavour dependent Gaussian width for the unpolarized fragmentation function, but we will refrain from doing so and stick to our simple picture.

It is instructive to compare our result (11) to the values extracted in Ref. [33] under certain assumptions from EMC data [42] on the so-called Cahn effect [43]. There, $p_{\text {unp }}^{2}=0.25 \mathrm{GeV}^{2}$ and $K_{D_{1}}^{2}=0.20 \mathrm{GeV}^{2}$ were found. With these numbers one obtains a $\left\langle P_{h \perp}(z)\right\rangle$ which describes the HERMES data almost as well as the direct fit in Eq. (11) — Fig. (2). (Notice that in the formalism of Ref. [33] the expression (10) holds approximately upon the neglect of terms of $\mathcal{O}\left(k_{\perp}^{2} / Q^{2}\right)$.) Thus, we are lead to the encouraging conclusion that the Gaussian ansatz for $f_{1}^{a}\left(x, \mathbf{p}_{T}^{2}\right)$ and $D_{1}^{a}\left(z, \mathbf{K}_{T}^{2}\right)$ is compatible with SIDIS data from HERMES [6] and that the ansatz and the numerical values for the Gaussian widths are supported qualitatively by the analysis [33] of EMC data [42] on the Cahn effect.

Unlike in the case of the unpolarized fragmentation and distribution functions, it is not possible to fix the Gaussian width of the Sivers function from SIDIS data. As the HERMES data [9] show a non-zero Sivers effect, of course, the parameter $p_{\text {Siv }}^{2}$ cannot be zero [72]. However, there is also a non-trivial upper bound for $p_{\text {Siv }}^{2}$ due to positivity conditions. The positivity bound for the Sivers distribution function reads [44]

$$
\frac{\left|\mathbf{p}_{T}\right|}{M_{N}}\left|f_{1 T}^{\perp a}\left(x, \mathbf{p}_{T}^{2}\right)\right| \leq f_{1}^{a}\left(x, \mathbf{p}_{T}^{2}\right) .
$$

If we demand the inequality (12) to be satisfied in the Gaussian model (5) at any value of $x$ and for all $\left|\mathbf{p}_{T}\right|$, then the following necessary and sufficient condition must hold

$$
\left(\frac{f_{1 T}^{\perp a}(x)}{f_{1}^{a}(x)}\right)^{2} \leq \frac{2 e M_{N}^{2}}{p_{\mathrm{unp}}^{2}} R(1-R) \quad \text { where } \quad R \equiv \frac{p_{\mathrm{siv}}^{2}}{p_{\mathrm{unp}}^{2}} .
$$

This means that the Gaussian width is bound from below and above as follows

$$
\frac{1}{2}-\sqrt{\frac{1}{4}-\frac{p_{\mathrm{unp}}^{2}}{2 e M_{N}^{2}}\left(\frac{f_{1 T}^{\perp a}(x)}{f_{1}^{a}(x)}\right)^{2}} \leq R \leq \frac{1}{2}+\sqrt{\frac{1}{4}-\frac{p_{\mathrm{unp}}^{2}}{2 e M_{N}^{2}}\left(\frac{f_{1 T}^{\perp a}(x)}{f_{1}^{a}(x)}\right)^{2}} .
$$

In particular, if $f_{1 T}^{\perp a}(x) \neq 0$ as the data tell us, then $0<R<1$ must be satisfied. Thus, we see that $p_{\text {Siv }}^{2}$ is restricted to the range

$$
0<p_{\mathrm{Siv}}^{2}<0.33 \mathrm{GeV}^{2} .
$$

It is worthwhile stressing that there is a bound for $p_{\text {Siv }}^{2}$ even if $f_{1 T}^{\perp a}(x)$ is very small.

For later convenience let us derive from (12) bounds for the transverse moment of the Sivers function. From the Gaussian version (13) of the positivity bound we obtain immediately

$$
\left(\frac{f_{1 T}^{\perp(1) a}(x)}{f_{1}^{a}(x)}\right)^{2} \leq \frac{e p_{\mathrm{unp}}^{2}}{2 M_{N}^{2}} R^{3}(1-R) \leq \frac{e p_{\mathrm{unp}}^{2}}{2 M_{N}^{2}} \frac{3^{3}}{4^{4}} .
$$

Notice that from (12) one also can derive a model-independent bound as follows. Using

$$
\left|\int \mathrm{d}^{2} \mathbf{p}_{T} \frac{\mathbf{p}_{T}^{2}}{2 M_{N}^{2}} f_{1 T}^{\perp a}\left(x, \mathbf{p}_{T}^{2}\right)\right| \leq \int \mathrm{d}^{2} \mathbf{p}_{T} \frac{\mathbf{p}_{T}^{2}}{2 M_{N}^{2}}\left|f_{1 T}^{\perp a}\left(x, \mathbf{p}_{T}^{2}\right)\right| \leq \int \mathrm{d}^{2} \mathbf{p}_{T} \frac{\left|\mathbf{p}_{T}\right|}{2 M_{N}} f_{1}^{a}\left(x, \mathbf{p}_{T}^{2}\right)
$$


and the definition (7) we obtain

$$
\left|f_{1 T}^{\perp(1) a}(x)\right| \leq \frac{\left\langle p_{T}\right\rangle_{\mathrm{unp}}}{2 M_{N}} f_{1}^{a}(x) .
$$

In the derivation of this bound no use was made of any transverse momentum model. Therefore, it must be valid in any model. In fact, by evaluating $\left\langle p_{T}\right\rangle_{\text {unp }}$ in the Gaussian model (7), we find that the model bound (16) is stronger than (17), i.e. if the transverse moment of the Sivers functions satisfies (16) then it fullfils automatically also (17).

When using the inequality (13) in our approach with the Gaussian widths assumed to be $x$-independent, it is understood that the ratio of the Sivers function (or its moment) to $f_{1}^{a}(x)$ is to be evaluated at that point in $x$, where it takes its maximal value.

The present SIDIS data with their sizeable error bars $[9,10]$ do not constrain fits for $f_{1 T}^{\perp a}$ for the separate flavours $a=u, d, \bar{u}$ and $\bar{d}$ assuming the effects of heavier quarks to be negligible. In fact, in Ref. [33] where this has been attempted all fitted distributions but $f_{1 T}^{\perp u}$ were found consistent with zero. In this situation it appears appealing to invoke additional theoretical constraints. In particular, here we will use predictions from the QCD limit of a large number of colours $N_{c}$.

In this limit the nucleon appears as $N_{c}$ quarks bound by a mean field [45], which exhibits certain spin-flavour symmetries [46]. By exploring these symmetry properties it was proven in a model independent way that in the large- $N_{c}$ limit [41]

$$
\underbrace{\left|\left(f_{1 T}^{\perp u}-f_{1 T}^{\perp d}\right)\left(x, \mathbf{p}_{T}^{2}\right)\right|}_{=\mathcal{O}\left(N_{c}^{3}\right)} \gg \underbrace{\left|\left(f_{1 T}^{\perp u}+f_{1 T}^{\perp d}\right)\left(x, \mathbf{p}_{T}^{2}\right)\right|}_{=\mathcal{O}\left(N_{c}^{2}\right)},
$$

or, equivalently,

$$
f_{1 T}^{\perp u}\left(x, \mathbf{p}_{T}^{2}\right)=-f_{1 T}^{\perp d}\left(x, \mathbf{p}_{T}^{2}\right) \text { modulo } 1 / N_{c} \text { corrections. }
$$

The relations $(18,19)$ are expected to be valid within their accuracy in the region of not too small and not too large $x$ satisfying $x N_{c}=\mathcal{O}\left(N_{c}^{0}\right)$. Similar relations hold for the Sivers antiquark distributions[73].

Inspired by the large- $N_{c}$ relation (19) we choose the following ansatz:

$$
x f_{1 T}^{\perp(1) u}(x)=-x f_{1 T}^{\perp(1) d}(x)=A x^{b}(1-x)^{5},
$$

and set $f_{1 T}^{\perp(1) \bar{q}}(x)$, as well as the Sivers distributions of heavier quarks, to zero. The shape of the Sivers function at large $x$ cannot be constrained by the data $[9,10]$. The large- $x$ behaviour $f_{1 T}^{\perp(1) q}(x) \propto(1-x)^{5}$ can be justified under certain assumptions - see [32] and references therein. However, one may consider it here as a mere model ansatz.

Given the size of the error bars of the present data the ansatz (20) and the above assumptions are not too restrictive. This was exemplified in Ref. [32] in a study of preliminary HERMES data [8] on the transverse-momentum-weighted Sivers SSA (3).

\section{EXTRACTION OF THE SIVERS FUNCTION FROM HERMES DATA}

In the previous Sections we fixed the parameter $K_{D_{1}}^{2}$ in Eq. (11), but all we have been able to do so far concerning $p_{\text {Siv }}^{2}$ was to constrain this parameter to the vague range in Eq. (15). However, the parameter $p_{\text {Siv }}^{2}$ appears explicitly only in the "Gaussian factor" $a_{\text {Gauss }}$ defined in Eq. (8). For illustrative purposes we evaluate this factor for the $\left\langle z^{2}\right\rangle \approx(0.4)^{2}$ of the HERMES experiment in the range (15) of the a priori possible value for $p_{\text {Siv }}^{2}$, and we observe that it is strongly constrained to be in the range

$$
0.72<a_{\text {Gauss }}<0.83 .
$$

Thus, as long as one is interested in extracting from the HERMES data [9] the transverse moment $f_{1 T}^{\perp(1) a}(x)$ under the assumption of the Gaussian ansatz (5), then the result is only affected to at most $\pm 10 \%$ by variations of the parameter $p_{\text {Siv }}^{2}$ within the poorly constrained range given in (15).

Note that as soon as we extract a result for $f_{1 T}^{\perp(1) a}(x)$, then from the inequality (16) we immediately obtain bounds for $p_{\text {Siv }}^{2}$ which are stronger than those given in (15). We proceed as follows. 
(a)

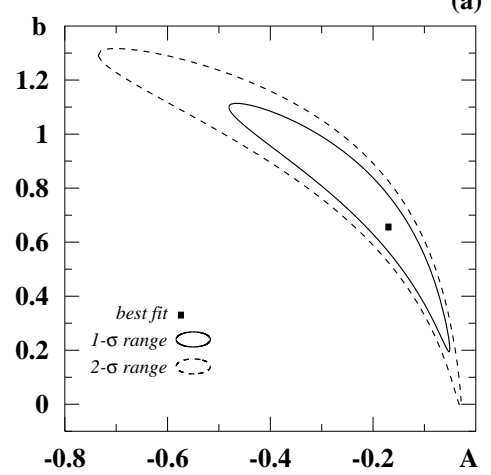

(b)

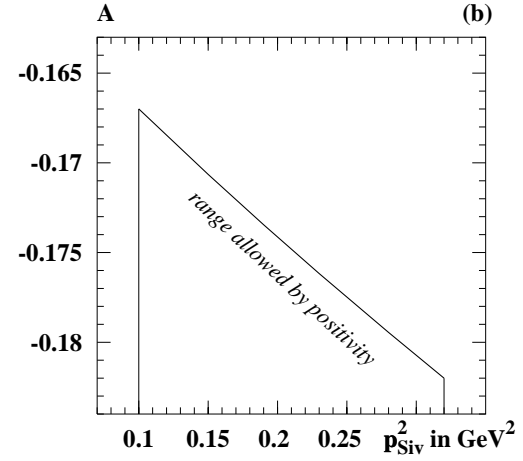

FIG. 3: a. The best fit and the respectively 1- and 2- $\sigma$ range for the parameters $A$ and $b$ in the ansatz (20) for the Sivers function. b. The dependence of the parameter $A$ on the Gaussian width $p_{\mathrm{Siv}}^{2}$ characterizing the transverse momentum distribution in the Sivers function in the Gaussian model (5). The parameter $b$ is practically $p_{\mathrm{Siv}^{2}}^{2}$-independent.
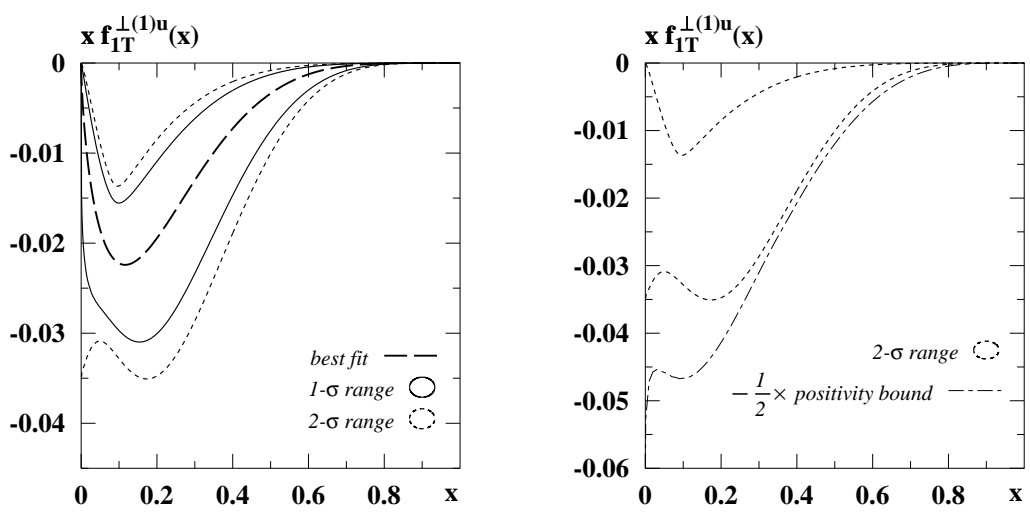

FIG. 4: a. The $u$-quark Sivers function $x f_{1 T}^{\perp(1) u}(x)$ as function of $x$, as extracted from the HERMES data [9]. Shown are the best fit, and its 1 - and 2- $\sigma$ regions. b. Here it is shown that the absolute value of the extracted Sivers function does not exceed half of the positivity bound in the Gaussian model in Eq. (16).
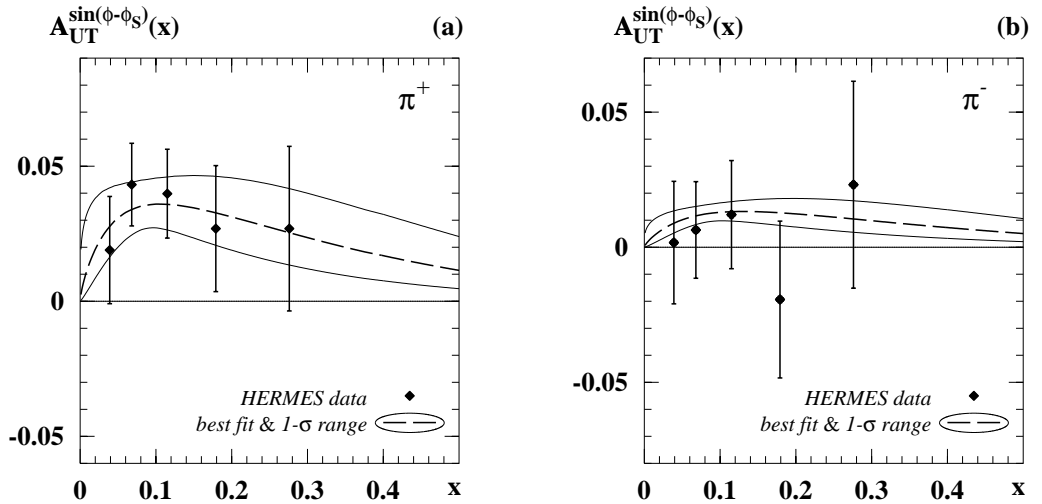

FIG. 5: The azimuthal SSA $A_{U T}^{\sin \left(\phi_{h}-\phi_{S}\right)}$ as function of $x$ for charged pions as obtained from the fit (23) in comparison to the final HERMES data [9]. 
We use the parameterizations $[50,51]$ for $f_{1}^{a}(x)$ and $D_{1}^{a}(z)$ at a scale of $2.5 \mathrm{GeV}^{2}$ which corresponds to the average $Q^{2}$ in the HERMES experiment. We insert the large- $N_{c}$ motivated ansatz $(20)$ in the expression for the $x$-dependent Sivers SSA in Eq. (8)

$$
A_{U T}^{\sin \left(\phi-\phi_{S}\right)}=(-2) \frac{\int \mathrm{d} z a_{\mathrm{Gauss}}(z) \sum_{a} e_{a}^{2} x f_{1 T}^{\perp(1) a}(x) D_{1}^{a}(z)}{\int \mathrm{d} z \sum_{a} e_{a}^{2} x f_{1}^{a}(x) D_{1}^{a}(z)}
$$

where we consider the $z$-dependence of the Gauss factor $a_{\text {Gauss }}$ and integrate within the cuts [9] of the HERMES experiment, $0.2 \leq z \leq 0.7$. We fix $K_{D_{1}}^{2}$ according to Eq. (11) and choose for the parameter $p_{\text {Siv }}^{2}$ a value out of the range (15) of the a priori possible values. Then we check whether for the chosen $p_{\text {Siv }}^{2}$ the extracted $f_{1 T}^{\perp(1) a}(x)$ does satisfy the inequality (16) within its $2-\sigma$-uncertainty. We obtain the following result which refers to a scale of about $2.5 \mathrm{GeV}^{2}$ :

$$
\begin{aligned}
x f_{1 T}^{\perp(1) u}(x)=-x f_{1 T}^{\perp(1) d}(x) & =-(0.17 \ldots 0.18) x^{0.66}(1-x)^{5} \\
\text { with } p_{\text {Siv }}^{2} & =(0.10 \ldots 0.32) \mathrm{GeV}^{2}
\end{aligned}
$$

Several comments are in order. The total $\chi^{2}$ is about 2.2 , i.e., the $\chi^{2}$ per degree of freedom is about 0.3 .

In Fig. 3a the $1-$ and $2-\sigma$ regions of the parameters $A$ and $b$ are shown for $p_{\text {Siv }}^{2}=0.20 \mathrm{GeV}^{2}$, which is the central value of $p_{\text {Siv }}^{2}$ in the range (24). The dependence of the parameter $b$ on $p_{\text {Siv }}^{2}$ is negligible. The response of the parameter $A$ to variations of $p_{\text {Siv }}^{2}$ is nearly linear - Fig. $3 \mathrm{~b}$.

The range (24) consists of those values of $p_{\text {Siv }}^{2}$ that are allowed by the general positivity inequality in the Gaussian model in Eq. (13). The data [9] do not allow us to constrain the parameter $p_{\text {Siv }}^{2}$ more accurately than that. However, it is satisfactory to observe how little the fit result for $f_{1 T}^{\perp(1) a}$ in Eq. (23) is affected by the fact that the parameter $p_{\text {Siv }}^{2}$ is only poorly constrained in the range (24).

In Fig. 4a we show the extracted $u$-quark Sivers function $x f_{1 T}^{\perp(1) u}(x)$. For the curve labeled as "best fit" we have chosen $p_{\text {Siv }}^{2}=0.20 \mathrm{GeV}^{2}$ which corresponds to the central value in the range (24) allowed by positivity requirements. For the minima (maxima) of the 1- and 2- $\sigma$ regions we have chosen the minimal (maximal) value of $p_{\text {Siv }}^{2}$ in the range (24). Thus, the displayed error bands contain both, the statistical error of the HERMES data [9] and the uncertainty due to the poorly constrained Gaussian width $p_{\text {Siv }}^{2}$ of the Sivers function in Eq. (24).

Notice that strictly speaking we neglected a low- $P_{h \perp}$ cut in Eq. (8) and one may wonder how large is the error we introduced in this way. With the results we obtained (neglecting such cuts) in Eqs. (11, 24) we find that taking this cut into account in the HERMES kinematics would change our results by about $1 \%$ (and about $2 \%$ at COMPASS to be discussed below). Thus, in the Gaussian model the neglect of the low- $P_{h \perp}$ cut is justified.

The absolute values of the extracted Sivers functions $f_{1 T}^{\perp(1) u}(x)=-x f_{1 T}^{\perp(1) d}(x)$ are restricted by the upper (Gaussian model) bound (16) given numerically by $0.23 f_{1}^{u}(x)$ or $0.23 f_{1}^{d}(x)$. Since $f_{1}^{d}(x)$ is smaller than $f_{1}^{u}(x)$ the bound is stronger for the $d$-quark. In Fig. $4 \mathrm{~b}$ we see that the extracted Sivers function well satisfies this bound, and we remark that it does not even exceed half of the general bound (17) within its $2-\sigma$ uncertainty.

Finally, in Fig. 5 we compare the Sivers SSA obtained on the basis of our fit (23) to the HERMES data [9]. Of course, in the SSA the effects of the (small) uncertainty of the parameter $A$ in (23) and the (sizeable) uncertainty of the parameter $p_{\mathrm{Siv}}^{2}$ in (24) cancel. Notably, the 1- $\sigma$ error band for the $\pi^{-}$SSA is much narrower than for the $\pi^{+}$SSA. This means that the $\pi^{-}$SSA is more sensitive to $1 / N_{c}$ corrections, i.e., to deviations from the ansatz (20). We will discuss this point in detail in the next Section.

As an intermediate summary we conclude that the HERMES data [9] are well compatible with the large- $N_{c}$ predictions (19) for the Sivers function [41] and that the fit (23) satisfies the positivity bounds [44]. Remarkably, the sign of the extracted Sivers function in Eq. (23) is in agreement with the physical picture discussed in [52]. We remark, however, that model calculations of the Sivers function [53-55] show no tendency to exhibit the large- $N_{c}$ pattern $(19)$.

\section{CROSS CHECKS: HERMES DATA ON $z$-DEPENDENCE \& COMPASS DATA}

In our fitting procedure we did not use the HERMES data [9] on the $z$-dependence of the Sivers SSA. These data could have been used as an additional constraint for the integrals of $x f_{1 T}^{\perp(1) a}(x)$ in the range $0.023<x<0.4$, which corresponds to the cuts in the HERMES experiment. This would have helped to improve the significance of the fit, considering that only few $x$-data points are available. Instead, let us use these data here as a valuable cross check of our approach. As the $z$-shape of the SSA is dictated by the unpolarized fragmentation function $D_{1}^{a}(z)$ and the $z$-dependence of the Gaussian factor $a_{\text {Gauss }}$ in Eq. (8), this is not only a cross check for the extracted Sivers function (23), but it also tests the Gauss ansatz (5), the consistency of the choice of parameters $(11,24)$, and the large- $N_{c}$ 

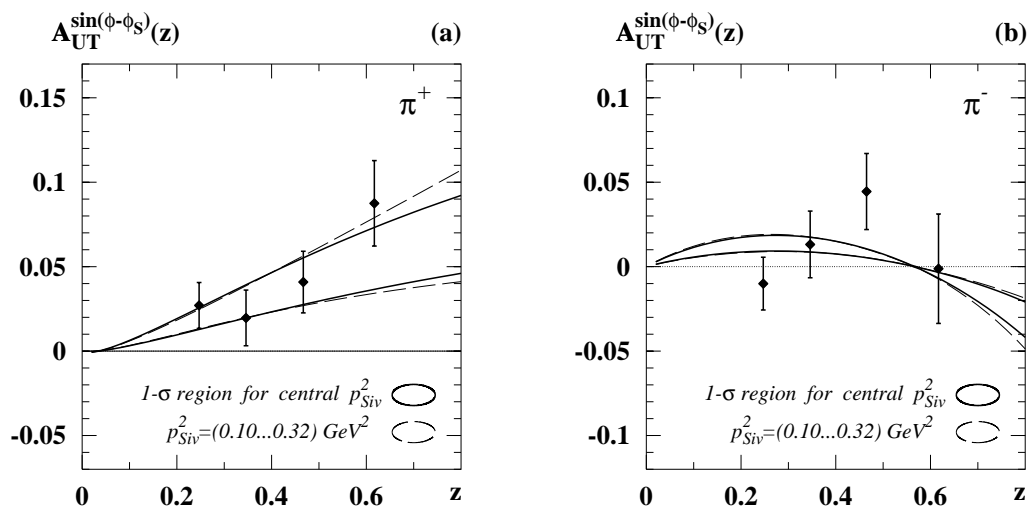

FIG. 6: $A_{U T}^{\sin \left(\phi_{h}-\phi_{S}\right)}$ as function of $z$. The data are from the HERMES experiment [9]. The curves show the 1- $\sigma$ variation of our large- $N_{c}$ constrained fit (23) for the Sivers function, and the impact of varying the Gaussian width $p_{\mathrm{Siv}}^{2}$ of the Sivers function in the range (24). The z-dependent data were not used for the fit, i.e., the comparison serves as a cross check for our results.

ansatz $(19,20)$ itself. In Fig. 6 we confront our fit result (23) with the $z$-dependent HERMES data on the Sivers SSA [9]. We observe a satisfactory agreement. Notice that the impact of the poorly constrained Gaussian width $p_{\text {Siv }}^{2}$ of the Sivers function (24) is marginal.

The smallness of the $\pi^{-}$SSA can be explained as follows. Since $f_{1 T}^{\perp u}=-f_{1 T}^{\perp d}$ in the large- $N_{c}$ limit, the asymmetry $A_{U T}^{\sin \left(\phi_{h}-\phi_{S}\right)}\left(\pi^{-}\right) \propto\left(\frac{4}{9} D_{1}^{\text {unf }}-\frac{1}{9} D_{1}^{\text {fav }}\right)$, where $D_{1}^{\text {fav }}=D_{1}^{d \rightarrow \pi^{-}}=D_{1}^{\bar{u} \rightarrow \pi^{-}}=D_{1}^{u \rightarrow \pi^{+}}$, etc. $\left(D_{1}^{\text {unf }}=D_{1}^{u \rightarrow \pi^{-}}=D_{1}^{d \rightarrow \pi^{+}}\right.$, etc.), denotes the so-called favoured (unfavoured) fragmentation function. At any $z$ we have the inequality $D_{1}^{\mathrm{unf}}(z)<D_{1}^{\mathrm{fav}}(z)$. However, due to the weighting by the square of the quark electric charges, the effects of the smaller "unfavoured" and the larger "favoured" fragmentation function become comparable and tend to cancel each other. As a result the SSA for $\pi^{-}$appears rather small, becoming zero around $z=0.56$ (for the parameterization in [51] at $Q^{2}=2.5 \mathrm{GeV}^{2}$ ). The tendency for cancellation persists with inclusion of the $1 / N_{c}$ corrections, which, however, shift the position of the zero - see Sec. VI.

Results from the COMPASS experiment [10] yield an equally important confirmation for the large $N_{c}$ picture of the Sivers function. In the COMPASS experiment a solid polarized ${ }^{6} \mathrm{LiD}$ target $[56,57]$ was used. Neglecting, to a first approximation, nuclear binding effects and using isospin symmetry, we observe that for the deuterium Sivers distribution function we have $f_{1 T}^{u / D} \approx f_{1 T}^{u / p}+f_{1 T}^{u / n} \approx f_{1 T}^{u}+f_{1 T}^{d}$, and analogously for $d, \bar{q}$, etc. Thus, the deuterium target is sensitive to the flavour combination which is suppressed in the large- $N_{c}$ limit, see (18), and for which our ansatz (20) yields exactly zero. This is in agreement with the present COMPASS data which shows a Sivers effect from deuterium target compatible with zero within error bars [10].

\section{WHERE ARE SIVERS ANTIQUARKS \& 1/ $N_{c}$ CORRECTIONS?}

In the ansatz (20) we neglected the Sivers distributions for antiquarks and for the strange and heavier quarks. The neglect of strange and heavier quarks is probably a good assumption at the present stage of art. However, neglecting the Sivers antiquarks need not be such a good approximation. This can be seen from the unpolarized distribution functions, since in the region of $x \lesssim 0.15$ the distribution of unpolarized $\bar{d}$-quarks reaches $25 \%$ and more of the unpolarized $d$-quark distribution. In fact, this is precisely the $x$-region where HERMES [9] observes the most significant Sivers effect (for $\pi^{+}$) - see Fig. 5 .

In order to gain a rough idea of the possible uncertainty introduced by neglecting the Sivers antiquark distributions, we make two simple models for the Sivers $\bar{u}$ - and $\bar{d}$-distributions, while keeping the quark distributions at the value given by our fit result (23). Model I is that Sivers $\bar{q}$-distributions are just $\pm 25 \%$ of the corresponding Sivers quark distributions. This assumption may be an overestimate in the region of larger $x$. Therefore, in model II we set the ratio of each Sivers $\bar{q}$ - to Sivers $q$-distribution to be the same as for the unpolarized distributions. Thus, we will explore the effects of assuming each of the following models for the $\bar{q}$-Sivers distributions:

$$
f_{1 T}^{\perp(1) \bar{q}}(x)=\epsilon(x) f_{1 T}^{\perp(1) q}(x), \quad \text { with } \quad \epsilon(x)= \pm \begin{cases}0.25=\text { const } & \text { model I }, \\ \frac{\left(f_{1}^{\bar{u}}+f_{1}^{\bar{d}}\right)(x)}{\left(f_{1}^{u}+f_{1}^{d}\right)(x)} & \text { model II } .\end{cases}
$$



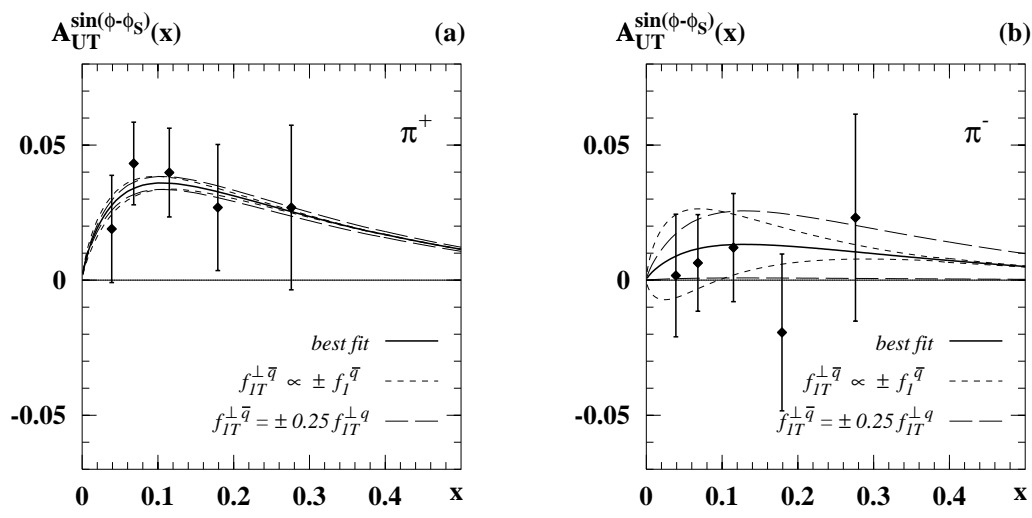

FIG. 7: $A_{U T}^{\sin \left(\phi_{h}-\phi_{S}\right)}$ as a function of $x$. The data are from the HERMES experiment [9]. The curves show the effect of modifying the result of large- $N_{c}$ constrained fit (23) by using the Sivers antiquark distribution functions given in Eq. (25). The figures show that the data [9] have little sensitivity to the Sivers $\bar{q}$-distributions, so that the neglect of the antiquark distributions in the fit was justified.

Note that the particular ansatz for model II ensures compatibility with the large- $N_{c}$ limit, where $\left(f_{1}^{u}+f_{1}^{d}\right)(x) \sim$ $\mathcal{O}\left(N_{c}^{2}\right) \gg\left(f_{1}^{u}-f_{1}^{d}\right)(x) \sim \mathcal{O}\left(N_{c}\right)$. Thus, our model Sivers antiquarks satisfy large- $N_{c}$ relations analogous to $(18,19)$. We also automatically preserve the sum rule [64] (see also [32]),

$$
\sum_{a=g, u, d, \ldots} \int \mathrm{d} x f_{1 T}^{\perp(1) a}(x)=0 .
$$

In the large- $N_{c}$ limit the gluon Sivers distribution is suppressed with respect to the quark one [32]. Our models (25) satisfy the inequality corresponding to (17). Thus, being compatible with all theoretical constraints we are presently aware of, the models (25) are well suited for our purposes.

In Fig. 7 we show the modest effect of the Sivers $\bar{q}$-distributions as a function of $x$. The effect is quite small for the $\pi^{+}$SSA, and well within the experimental errors. The effect is more pronounced for the $\pi^{-}$SSA, but still remains within the experimental errors. The effect of Sivers antiquarks on the $z$-dependence of the Sivers SSA is less visible, and we refrain from showing analog plots. As here the $x$-dependence is integrated over, the entire effects amount of altering the overall normalization of the SSA without qualitative and with only small quantitative changes to the picture in Fig. 6.

Thus, we conclude that even sizeable Sivers antiquark distributions, as modeled in Eq. (25), cannot be resolved within the error bars of the present data [9]. This justifies a posteriori the neglect of Sivers $\bar{q}$-distribution functions in our fit ansatz (20) here or in Ref. [32]. In this way we confirm also the observation made in Ref. [33]. There an attempt was made to extract $f_{1 T}^{\perp a}$ for the separate flavours $a=u, d, \bar{u}$ and $\bar{d}$, and the Sivers- $\bar{q}$ distributions were found to be consistent with zero with large uncertainties.

The difficulty to access antiquark distribution functions in SIDIS data is more general than the example encountered here. For example, in longitudinal double spin asymmetries $A_{L L} \propto \sum_{a} e_{a}^{2} g_{1}^{a}(x) D_{1}^{a}(z)$ it is presently [58] not possible to resolve a flavour asymmetry in the helicity sea as sizeable as predicted in the chiral quark-soliton model [59], namely larger [60] than the known flavour asymmetry in the unpolarized sea $\left(g_{1}^{\bar{u}}-g_{1}^{\bar{d}}\right)(x)>\left|\left(f_{1}^{\bar{u}}-f_{1}^{\bar{d}}\right)(x)\right|$. As in the case of the much better known distribution functions $f_{1}^{a}(x)$ and $g_{1}^{a}(x)$ (see [61] for the specific example of helicity sea flavour asymmetry from the chiral quark-soliton model), it is necessary to study the Drell-Yan process in order to learn more about Sivers antiquark distribution functions.

Next let us address the $1 / N_{c}$-corrections. In our ansatz (20) we took literally the prediction from the large- $N_{c}$ limit [41] in Eq. (19), disregarding corrections which are generically of $\mathcal{O}\left(1 / N_{c}\right) \approx 30 \%$. In order to have an idea of the effect of these corrections, let us assume that the flavour singlet Sivers distribution is not exactly zero but suppressed by exactly a factor of $1 / N_{c}$ with respect to the flavour non-singlet combination according to Eq. (18). That is,

$$
\left|\left(f_{1 T}^{\perp(1) u}+f_{1 T}^{\perp(1) d}\right)(x)\right| \stackrel{!}{=} \pm \frac{1}{N_{c}}\left(f_{1 T}^{\perp(1) u}-f_{1 T}^{\perp(1) d}\right)(x),
$$

where we use $f_{1 T}^{\perp(1) u}(x), f_{1 T}^{\perp(1) d}(x)$ from Eq. (23) and set $N_{c}=3$. Of course, it would be naive to expect that the different flavour combinations behave precisely as in Eq. (27). However, the scenario in Eq. (27) is by construction well suited to indicate the order of magnitude of the effect. In fact, when considered from such a qualitative point of view, large- $N_{c}$ relations for parton distributions are observed to be well satisfied in nature [62]. 

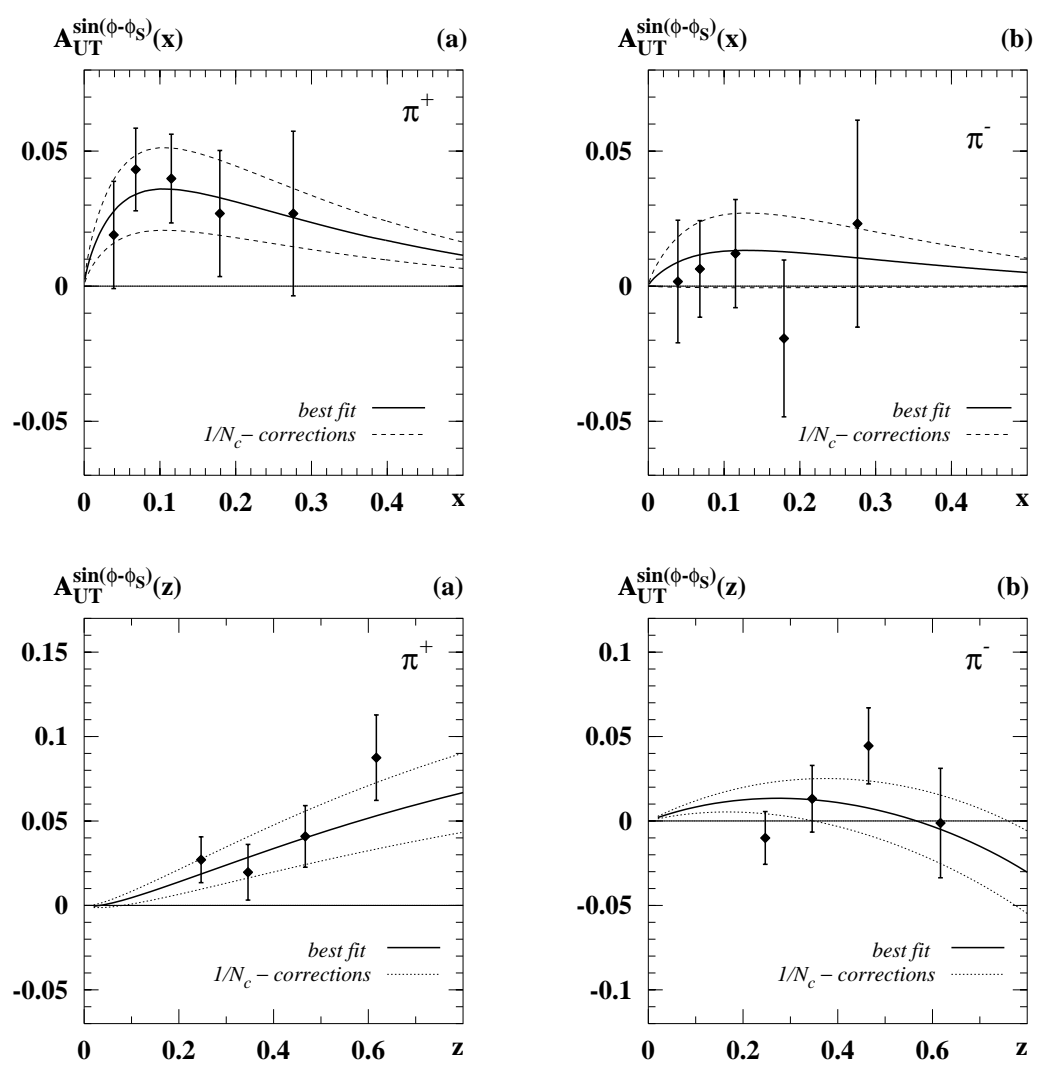

FIG. 8: a and b. $A_{U T}^{\sin \left(\phi_{h}-\phi_{S}\right)}$ as function of $x$. The data are from the HERMES experiment [9]. The curves show our best fit (23) for the Sivers function, and the effect of $1 / N_{c}$-corrections as modeled in Eq. (27). The figures show that the statistical uncertainty of the data [9] is of comparable magnitude as the $1 / N_{c}$-corrections. c and d. The same as in Figs. $8 \mathrm{a}$ and $8 b$ but for the $z$-dependence of the Sivers SSA.
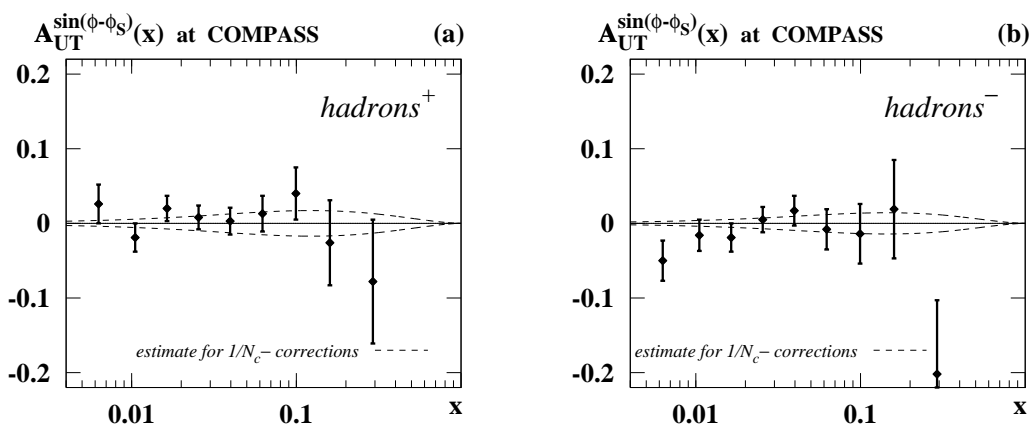

FIG. 9: The Sivers SSA at COMPASS. The theoretical curves indicate the order of magnitude of the effect according to the rough model for $1 / N_{c}$-corrections in Eq. (27). Thus, taking the large- $N_{c}$ counting rule (18) into account we conclude that the HERMES [10] and COMPASS [10] data are compatible with each other.

Note that (27) is compatible with positivity (17). However, in order to comply with the sum rule (26), we must introduce a gluon Sivers distribution function equal in magnitude but of opposite sign to the quark flavour singlet Sivers distribution.

Fig. 8 shows the effect of $1 / N_{c^{-}}$corrections as modeled in Eq. (27). The positive (negative) sign in Eq. (27) corresponds to the upper (lower) curves in Figs. 8a and 8b. What we obtained in this way is an "error band" of much the same size as the 1- $\sigma$ uncertainty of our fit in Fig. 5. A look at the $z$-dependence of the Sivers SSA fully confirms these findings - see Figs. $8 \mathrm{c}$ and $8 \mathrm{~d}$. When the HERMES $z$-cuts are used, $\left\langle a_{\text {Gauss }} D_{1}^{\text {fav }}\right\rangle \approx 2\left\langle a_{\text {Gauss }} D_{1}^{\text {unf }}\right\rangle$ to a good accuracy with the parameterization [51]. If this were exactly the case, then for the minus sign in Eq. (27) 
the $\pi^{-}$Sivers SSA would become exactly zero, as shown in Fig. 8b. More generally, we see that the vanishing of the $\pi^{-}$asymmetry at some value of $z$ is a feature which is robust against $1 / N_{c}$-corrections (and antiquark effects). The precise position of this zero, however, is very sensitive to $1 / N_{c}$-corrections - Fig. $8 \mathrm{~d}$.

On a deuteron target, the leading $1 / N_{c}$ prediction gives zero for the SSA, so that the $1 / N_{c}$ corrections are all that remain. Assuming for simplicity that the positive and negative hadrons identified at COMPASS are mainly pions, we obtain in our rough model (27) for $1 / N_{c^{-}}$-corrections the results shown in Fig. 9. The positive asymmetries are due to choosing the positive sign in Eq. (27). Clearly, we see that the COMPASS data [10] are compatible with the large- $N_{c}$ corrections being of a magnitude compatible with out model.

Thus, the reason why our large- $N_{c}$ approach works here, is due to the fact that current precision of the first experimental data $[9,10]$ is comparable to the theoretical accuracy of the large- $N_{c}$ relation (19). This is illustrated in a different way by Fig. 10 which shows that a variation of the best fit (23) of $\pm 30 \%$ corresponding to the generic size of $1 / N_{c}$ corrections is of similar size and shape as the 1- $\sigma$ region of the fit. In other words, $1 / N_{c}$ corrections (and antiquark effects) cannot be resolved within the error bars of the data $[9,10]$. In future, with increasing precision of the data, it will certainly be necessary to refine the fit ansatz (20) to include $1 / N_{c}$ corrections and antiquarks. In fact we have found that our fit (23) to the final HERMES data [9] is also compatible with the most recent and substantially more precise preliminary HERMES data given in [11].

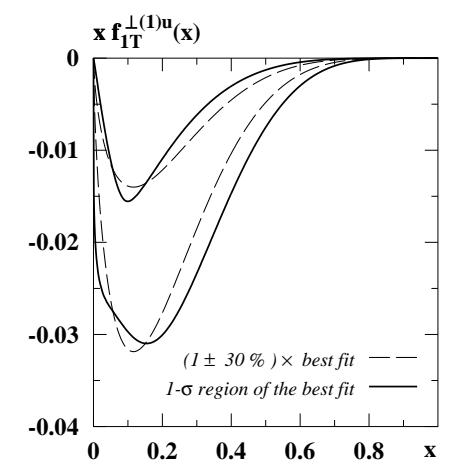

FIG. 10: The 1- $\sigma$ region of our best fit for $f_{1 T}^{\perp(1) u}(x)$ in comparison to a variation of $\pm 30 \%$ of our best fit (23), which roughly corresponds to the generic size of $1 / N_{c}$ corrections.

\section{FURTHER TESTS OF THE GAUSSIAN ANSATZ IN SIDIS}

We have seen that the Gaussian model (5) for the distribution of the transverse parton momenta provides a satisfactory effective description of the HERMES data on the average transverse momentum of pions produced in SIDIS [6]. In order to increase our faith into the applicability of the Gaussian model and — what is equally important - to find out its limitations, it is necessary to make further tests using, e.g., the available HERMES data both on polarized and unpolarized SIDIS. Although the true prediction in QCD, or any other field theory, is that there is approximately a power-law fall off at large transverse momentum, it can well be that a Gaussian dependence can be a useful approximation for transverse momenta that are low with respect to the relevant hard scale. This, in fact, corresponds to the situation in the HERMES experiment [9] where $\left\langle P_{h \perp}\right\rangle \sim 0.4 \mathrm{GeV} \ll \sqrt{\left\langle Q^{2}\right\rangle} \sim 1.5 \mathrm{GeV}$.

In this context it would be interesting to study the average transverse momentum square $\left\langle P_{h \perp}^{2}(z)\right\rangle$ of the produced hadrons given by (actually this relation is of more general character [63] and manifestly valid in the Gauss ansatz)

$$
\left\langle P_{h \perp}^{2}(z)\right\rangle=z^{2} p_{\mathrm{unp}}^{2}+K_{D_{1}}^{2} .
$$

In particular, $\left\langle P_{h \perp}(z)\right\rangle$ and $\left\langle P_{h \perp}^{2}(z)\right\rangle$ are related to each other in the Gaussian model by

$$
\left\langle P_{h \perp}(z)\right\rangle^{2} \stackrel{\text { Gauss }}{=} \frac{\pi}{4}\left\langle P_{h \perp}^{2}(z)\right\rangle
$$

To test the assumption of flavour independence, it would be useful to study $\left\langle P_{h \perp}(z)\right\rangle$ and $\left\langle P_{h \perp}^{2}(z)\right\rangle$ separately for $\pi^{+}$, $\pi^{0}, \pi^{-}$or the kaons. Finally, one could study the average transverse momentum of different hadrons averaged over $z$ but as function of the respective $x$-bin, in order to test the assumption of an $x$ - and flavour independent Gaussian width of the unpolarized distribution function.

Given the unsatisfactory situation with the poorly constrained Gaussian width of the Sivers function, it is of importance to further constrain this parameter by means of data — in particular on the Sivers SSA as function of $P_{h \perp}$. Appropriate HERMES data for this as well as the following suggestion are in principle available [8, 9].

Let us define the following "Sivers-mean-transverse-momentum":

$$
\left\langle P_{h \perp}(z)\right\rangle_{\mathrm{Siv}}=\frac{\sum_{i} P_{h \perp, i} \sin \left(\phi_{i}-\phi_{S, i}\right)\left[N^{\uparrow}\left(\phi_{i} ; \phi_{S, i}\right)-N^{\downarrow}\left(\phi_{i} ; \phi_{S, i}+\pi\right)\right]}{\sum_{i} \sin \left(\phi_{i}-\phi_{S, i}\right)\left[N^{\uparrow}\left(\phi_{i} ; \phi_{S, i}\right)-N^{\downarrow}\left(\phi_{i} ; \phi_{S, i}+\pi\right)\right]} \stackrel{\text { Gauss }}{=} \frac{2}{\sqrt{\pi}} \sqrt{z^{2} p_{\mathrm{Siv}}^{2}+K_{D_{1}}^{2}} .
$$


One can read off the result in the Gaussian model by comparing Eqs. (3) and (8). Note that $\left\langle P_{h \perp}(z)\right\rangle_{\text {Siv }}$ is of the same structure as its "unpolarized analog" $\left\langle P_{h \perp}(z)\right\rangle$ in Eq. (7) up to the coefficient $2 / \sqrt{\pi}$. From a simultaneous analysis of the Sivers SSA weighted with and without a power of transverse hadron momentum $\left\langle P_{h \perp}(z)\right\rangle_{\text {Siv }}$ could be determined with a relative accuracy comparable to that of the Sivers SSA.

An indirect but important test of the Gaussian ansatz for the Sivers function can be made on the basis of the analyses reported here and in [32]. In Ref. [32] the preliminary HERMES data [8] on the transverse-momentumweighted Sivers SSA (3) were used in order to directly extract $f_{1 T}^{\perp(1) a}(x)$ without resorting to any model for the transverse parton momenta. Here we have used the final HERMES data [9] to extract $f_{1 T}^{\perp(1) a}(x)$ under the assumption of the Gaussian model (5). Thus, if the Gaussian model works, then the two extractions must yield the same result.

In [32] the same set of assumptions (large- $N_{c}$ for $q$, neglect of $\bar{q}$, etc.) was used as here. However, instead of determining the 1- $\sigma$ region, two different ansätze were explored: One ansatz is as in (20), and another is as in (20) but with fixed $b=1$. In Fig. 11 we see that these fits from

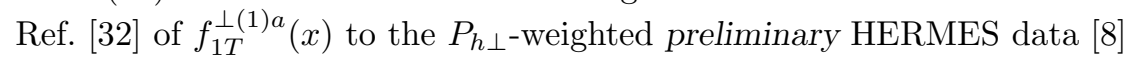
are compatible within 1- $\sigma$ with the fit to the final HERMES data on the Sivers SSA weighted without a power of $P_{h \perp}[9]$.

This observation indicates that the Gaussian ansatz for the Sivers function is compatible with the HERMES data within the statistical accuracy of the data [8,9]. Given the preliminary status of the data [8] it is not possible to draw a stronger conclusion from this observation at the present stage. In fact, the HERMES Collaboration does not recommend [65] the use of the preliminary data [8] since they are not corrected for acceptance effects specific to the

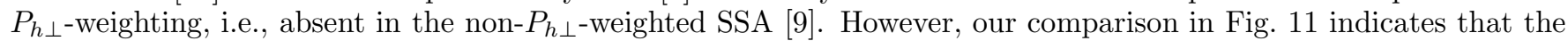
systematic error due to these effects is less dominant than the statistical uncertainty of the data [8,9].

Another important test for the Gaussian model and for the fit result (23) could use the HERMES data (and possible future data from COMPASS and Jlab) on the $\pi^{0}$ Sivers SSA. Preliminary data on the $\pi^{0}$ SSA with large statistical uncertainties were shown in [8]. Taking into account the 1- $\sigma$ uncertainty of the fit (23) we obtain the results shown in Fig. 12. Worthwhile commenting is the $z$-dependence of the $\pi^{0}$-asymmetry. Since the unpolarized fragmentation functions of light quarks and antiquarks into $\pi^{0}$ are the same (and since we neglect the effects of strange and heavier quarks $), D_{1}^{a}(z)$ completely cancel out from the SSA, so that $A_{U T}^{\sin \left(\phi-\phi_{S}\right)}\left(z, \pi^{0}\right) \propto a_{\text {Gauss }}(z)$. Thus the $z$-shape of the neutral pion SSA is entirely predicted by the Gaussian model. (This remains true even if one considers effects of $P_{h \perp}$ cuts. The effect of the small resolution cut applied at HERMES or COMPASS [9, 10] is negligible in Fig. 12, see also Sec. IV.) A precise measurement of the $z$-dependence of the $\pi^{0}$ Sivers effect could therefore also help to test the Gaussian Ansatz. Alternatively, one may combine appropriately $\pi^{+}$and $\pi^{-}$data and use isospin symmetry to arrive at the same information. This may yield results with better statistical accuracy in the HERMES experiment [69].
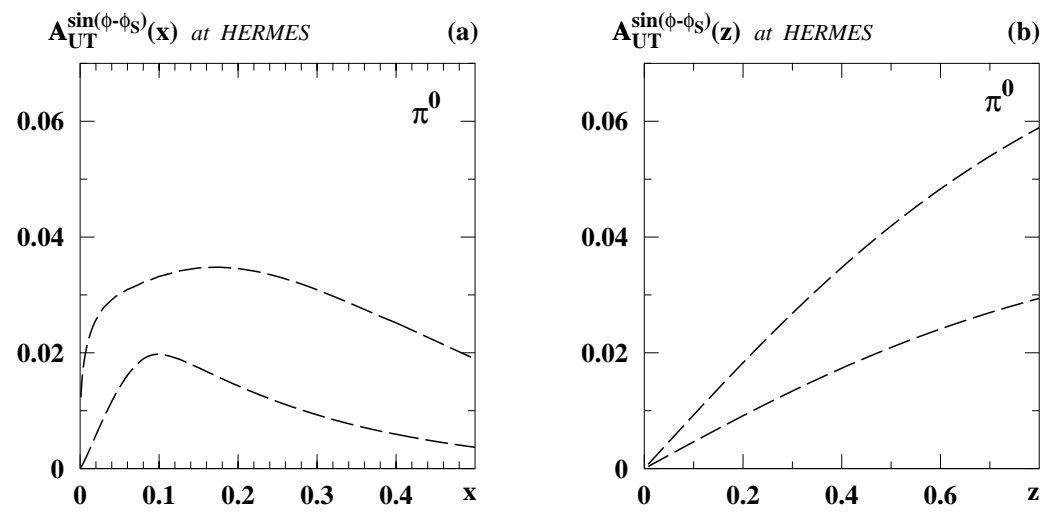

FIG. 12: The Sivers SSA for neutral pions at HERMES as function of $x$ and $z$, respectively, as predicted on the basis of the fit result (23). The error band arises from the 1- $\sigma$ uncertainty of the fit (23). 


\section{CONCLUSIONS}

In this work we have extracted the transverse moment $f_{1 T}^{\perp(1) a}$ of the Sivers function from SIDIS HERMES data [9] using a Gaussian model for the distribution of parton transverse momenta and employing predictions from the large$N_{c}$ limit [41] as an additional constraint. We have shown that the Gaussian model provides a reasonable description of HERMES data on the transverse momentum distribution of the hadrons produced in unpolarized SIDIS. We constrained the free parameters of the Gaussian model consistently by HERMES data, which however, does leave the Gaussian width of the Sivers distribution poorly constrained. Nevertheless, the data [9] well constrain a fit of the transverse moment of the Sivers function.

We have also shown that the HERMES and COMPASS data $[9,10]$ are compatible with each other and with predictions from the large- $N_{c}$ limit of QCD [41] within their statistical accuracy. We checked explictly that the effects of the simplifications we made are either negligibly small, as for example the neglect of the experimental resolution cuts on the transverse hadron momenta, or well within the statistical accuracy of the data, as the usage of large- $N_{c}$ constraints or the neglect of Sivers antiquark distributions. We provided cross and consistency checks for the fit result by studying the $z$-dependence of the HERMES data on the Sivers SSA [9], and made suggestions how to further test the applicability of the Gaussian model in SIDIS.

The main differences of our approach as compared to the similar works [33-35] are the use of the large- $N_{c}$ constraints, and the choice of a different model for transverse parton momenta and/or the way we fixed the respective parameters. Our fit is in qualitative agreement with extractions of the Sivers function [33-35] from the same [9] and from the more recent and more precise (but preliminary) HERMES data [11], see Ref. [70] for a detailed comparison.

Of particular interest are studies of the Sivers effect in the Drell-Yan (DY) process, because the Sivers function (and other "time-reversal-odd" distributions) are expected to obey an unusual universality property, namely to appear with opposite signs in SIDIS and in DY [18]. The experimental check of this prediction is a crucial test for the understanding of the Sivers effect within QCD. On the basis of an analysis of the preliminary HERMES data [8] it was shown [32] that this change of sign of the Sivers function could be checked in $p^{\uparrow} \pi^{-} \rightarrow l^{+} l^{-} X$ in the planned hadron-beam-mode of the COMPASS experiment [66], and in $p^{\uparrow} \bar{p} \rightarrow l^{+} l^{-} X$ in the proposed PAX experiment [67] (whose primary goal is to access the transversity distribution function in $p^{\uparrow} \bar{p}^{\uparrow} \rightarrow l^{+} l^{-} X$ [68]). Our study of the final HERMES data [9] presented here confirms the analysis of Ref. [32] solidifying the conclusions made there - as do the estimates reported in Refs. [34, 35] obtained from analyses of the most recent preliminary HERMES data [11]. Estimates for COMPASS, PAX and RHIC on the basis of the results obtained here will be presented elsewhere [71].

Acknowledgments. We thank Delia Hasch, Gunar Schnell and Alexei Prokudin for valuable discussions. We also thank Andy Miller for pointing out that the published data [9] are not final - contrary to the way we refer to them here - since the HERMES transverse target polarization run is not finished and more data are expected [11].

The work is partially supported by BMBF and DFG of Germany, the COSY-Jülich project, the Transregio BonnBochum-Giessen, and is part of the European Integrated Infrastructure Initiative Hadron Physics project under contract number RII3-CT-2004-506078. A.E. is supported by grants RFBR 03-02-16816 and DFG-RFBR 03-0204022. J.C.C. is supported in part by the U.S. D.O.E., and by a Mercator Professorship of DFG.

[1] G. Bunce et al., Phys. Rev. Lett. 36 1113, (1976).

[2] D. L. Adams et al., Phys. Lett. B 261, 201 and 264, 462 (1991), Z. Phys. C 56, 181 (1992).

[3] J. Felix, Mod. Phys. Lett. A 14, 827 (1999). M. Anselmino, Czech. J. Phys. 52, C13 (2002) [arXiv:hep-ph/0201150].

[4] H. Avakian [HERMES Collaboration], Nucl. Phys. Proc. Suppl. 79, 523 (1999).

[5] A. Airapetian et al. [HERMES Collaboration], Phys. Rev. Lett. 84, 4047 (2000) [arXiv:hep-ex/9910062], Phys. Rev. D 64, 097101 (2001) [arXiv:hep-ex/0104005].

[6] A. Airapetian et al. [HERMES Collaboration], Phys. Lett. B 562, 182 (2003) [arXiv:hep-ex/0212039].

[7] H. Avakian et al. [CLAS Collaboration], Phys. Rev. D 69, 112004 (2004) [arXiv:hep-ex/0301005]. E. Avetisyan, A. Rostomyan and A. Ivanilov [HERMES Collaboration], Proc. of DIS'2004, 13-18 April 2004, Strbské Pleso, Slovakia [arXiv:hep-ex/0408002].

[8] N. C. Makins [HERMES Collaboration], "Transversity Workshop", 6-7 Oct. 2003, Athens, Greece. R. Seidl [HERMES Collaboration], Proc. of DIS'2004, 13-18 April 2004, Štrbské Pleso, Slovakia. I. M. Gregor [HERMES Collaboration], Acta Phys. Polon. B 36, 209 (2005).

[9] A. Airapetian et al. [HERMES Collaboration], Phys. Rev. Lett. 94, 012002 (2005) [arXiv:hep-ex/0408013].

[10] V. Y. Alexakhin et al. [COMPASS Collaboration], Phys. Rev. Lett. 94, 202002 (2005) [arXiv:hep-ex/0503002]. P. Pagano [COMPASS Collaboration], arXiv:hep-ex/0501035.

[11] M. Diefenthaler, arXiv:hep-ex/0507013.

[12] J. C. Collins and D. E. Soper, Nucl. Phys. B 193, 381 (1981) [Erratum-ibid. B 213, 545 (1983)]. 
[13] X. D. Ji, J. P. Ma and F. Yuan, Phys. Rev. D 71, 034005 (2005) [arXiv:hep-ph/0404183], Phys. Lett. B 597, 299 (2004) [arXiv:hep-ph/0405085].

[14] J. C. Collins and A. Metz, Phys. Rev. Lett. 93, 252001 (2004) [arXiv:hep-ph/0408249].

[15] D. Boer and P. J. Mulders, Phys. Rev. D 57, 5780 (1998) [arXiv:hep-ph/9711485].

[16] D. W. Sivers, Phys. Rev. D 41, 83 (1990), Phys. Rev. D 43, 261 (1991).

[17] S. J. Brodsky, D. S. Hwang and I. Schmidt, Phys. Lett. B 530, 99 (2002) [arXiv:hep-ph/0201296]; Nucl. Phys. B 642, 344 (2002) [arXiv:hep-ph/0206259].

[18] J. C. Collins, Phys. Lett. B 536, 43 (2002) [arXiv:hep-ph/0204004].

[19] A. V. Belitsky, X. Ji and F. Yuan, Nucl. Phys. B 656, 165 (2003) [arXiv:hep-ph/0208038].

X. D. Ji and F. Yuan, Phys. Lett. B 543, 66 (2002) [arXiv:hep-ph/0206057].

D. Boer, P. J. Mulders and F. Pijlman, Nucl. Phys. B 667, 201 (2003) [arXiv:hep-ph/0303034].

[20] J. C. Collins, Nucl. Phys. B 396, 161 (1993) [arXiv:hep-ph/9208213].

[21] M. Anselmino, M. Boglione and F. Murgia, Phys. Lett. B 362, 164 (1995) [arXiv:hep-ph/9503290].

[22] M. Anselmino and F. Murgia, Phys. Lett. B 442, 470 (1998) [arXiv:hep-ph/9808426].

[23] U. D'Alesio and F. Murgia, Phys. Rev. D 70, 074009 (2004) [arXiv:hep-ph/0408092].

[24] M. Anselmino, M. Boglione, U. D'Alesio, E. Leader and F. Murgia, Phys. Rev. D 71, 014002 (2005) [arXiv:hep-ph/0408356].

[25] B. Q. Ma, I. Schmidt and J. J. Yang, Eur. Phys. J. C 40, 63 (2005) [arXiv:hep-ph/0409012].

[26] A. V. Efremov, K. Goeke and P. Schweitzer, Phys. Lett. B 522, 37 (2001) [arXiv:hep-ph/0108213] and 544, 389E (2002) [arXiv:hep-ph/0204056]; Eur. Phys. J. C 24, 407 (2002) [arXiv:hep-ph/0112166]; Nucl. Phys. A 711, 84 (2002); Acta Phys. Polon. B 33, 3755 (2002) [arXiv:hep-ph/0206267]. P. Schweitzer and A. Bacchetta, Nucl. Phys. A 732, 106 (2004) [arXiv:hep-ph/0310318].

[27] E. De Sanctis, W. D. Nowak and K. A. Oganessian, Phys. Lett. B 483, 69 (2000) [arXiv:hep-ph/0002091]; K. A. Oganessian, N. Bianchi, E. De Sanctis and W. D. Nowak, Nucl. Phys. A 689, 784 (2001) [arXiv:hep-ph/0010261].

[28] B. Q. Ma, I. Schmidt and J. J. Yang, Phys. Rev. D 66, 094001 (2002) [arXiv:hep-ph/0209114]; Phys. Rev. D 65, 034010 (2002) [arXiv:hep-ph/0110324].

[29] A. V. Efremov, K. Goeke and P. Schweitzer, Phys. Lett. B 568, 63 (2003) [arXiv:hep-ph/0303062].

[30] M. Anselmino, M. Boglione, U. D'Alesio, A. Kotzinian, F. Murgia and A. Prokudin, arXiv:hep-ph/0412316.

[31] A. V. Efremov, K. Goeke and P. Schweitzer, Czech. J. Phys. 55 A, 189 (2005) [arXiv:hep-ph/0412420].

[32] A. V. Efremov, K. Goeke, S. Menzel, A. Metz and P. Schweitzer, Phys. Lett. B 612, 233 (2005) [arXiv:hep-ph/0412353].

[33] M. Anselmino, M. Boglione, U. D’Alesio, A. Kotzinian, F. Murgia and A. Prokudin, Phys. Rev. D 71, 074006 (2005) [arXiv:hep-ph/0501196].

[34] M. Anselmino, M. Boglione, U. D’Alesio, A. Kotzinian, F. Murgia and A. Prokudin, arXiv:hep-ph/0507181.

[35] W. Vogelsang and F. Yuan, arXiv:hep-ph/0507266.

[36] A. Airapetian et al. [HERMES Collaboration], Phys. Lett. B 622, 14 (2005) [arXiv:hep-ex/0505042].

[37] P. J. Mulders and R. D. Tangerman, Nucl. Phys. B 461 (1996) 197 [Erratum-ibid. B 484 (1997) 538] [arXiv:hep$\mathrm{ph} / 9510301]$.

[38] A. Afanasev and C. E. Carlson, arXiv:hep-ph/0308163. A. Metz and M. Schlegel, Eur. Phys. J. A 22, 489 (2004) [arXiv:hepph/0403182]; Annalen Phys. 13, 699 (2004) [arXiv:hep-ph/0411118]. A. Bacchetta, P. J. Mulders and F. Pijlman, Phys. Lett. B 595 (2004) 309 [arXiv:hep-ph/0405154]. K. Goeke, A. Metz and M. Schlegel, Phys. Lett. B 618, 90 (2005) [arXiv:hep-ph/0504130].

[39] A. Bacchetta, U. D’Alesio, M. Diehl and C. A. Miller, Phys. Rev. D 70, 117504 (2004) [arXiv:hep-ph/0410050].

[40] I.e., distributions not integrated over the transverse parton momenta. For a discussion and careful definition of these objects in QCD we refer to: J. C. Collins, Acta Phys. Polon. B 34, 3103 (2003) [arXiv:hep-ph/0304122].

[41] P. V. Pobylitsa, arXiv:hep-ph/0301236.

[42] M. Arneodo et al. [European Muon Collaboration], Z. Phys. C 34 (1987) 277.

[43] R. N. Cahn, Phys. Lett. B 78 (1978) 269.

[44] A. Bacchetta, M. Boglione, A. Henneman and P. J. Mulders, Phys. Rev. Lett. 85, 712 (2000) [arXiv:hep-ph/9912490].

[45] E. Witten, Nucl. Phys. B 160, 57 (1979).

[46] A. P. Balachandran, V. P. Nair, S. G. Rajeev and A. Stern, Phys. Rev. D 27, 1153 and 2772E (1983).

E. Witten, Nucl. Phys. B 223, 433 (1983).

J. L. Gervais and B. Sakita, Phys. Rev. Lett. 52, 87 (1984).

A. V. Manohar, Nucl. Phys. B 248, 19 (1984).

D. Diakonov, V. Y. Petrov and P. V. Pobylitsa, Nucl. Phys. B 306, 809 (1988).

R. F. Dashen, E. Jenkins and A. V. Manohar, Phys. Rev. D 49, 4713 (1994) and 51, 2489E (1995) [arXiv:hep-ph/9310379].

[47] M. Anselmino, V. Barone, A. Drago and F. Murgia, Nucl. Phys. Proc. Suppl. 105, 132 (2002) [arXiv:hep-ph/0111044] and arXiv:hep-ph/0209073.

[48] P. V. Pobylitsa, arXiv:hep-ph/0212027.

[49] A. Drago, Phys. Rev. D 71, 057501 (2005) [arXiv:hep-ph/0501282].

[50] M. Glück, E. Reya and A. Vogt, Eur. Phys. J. C 5, 461 (1998) [arXiv:hep-ph/9806404].

[51] S. Kretzer, E. Leader and E. Christova, Eur. Phys. J. C 22, 269 (2001) [arXiv:hep-ph/0108055].

[52] M. Burkardt, Phys. Rev. D 66, 114005 (2002) [arXiv:hep-ph/0209179]; Nucl. Phys. A 735, 185 (2004) [arXiv:hep$\mathrm{ph} / 0302144]$.

[53] A. Bacchetta, A. Schäfer and J. J. Yang, Phys. Lett. B 578 (2004) 109 [arXiv:hep-ph/0309246].

[54] Z. Lu and B. Q. Ma, Nucl. Phys. A 741 (2004) 200 [arXiv:hep-ph/0406171]. 
[55] F. Yuan, Phys. Lett. B 575 (2003) 45 [arXiv:hep-ph/0308157].

[56] J. Ball et al., Nucl. Instrum. Meth. A 498, 101 (2003).

[57] S. Goertz, W. Meyer and G. Reicherz, Prog. Part. Nucl. Phys. 49, 403 (2002) [Erratum-ibid. 51, 309 (2003)].

[58] A. Airapetian et al. [HERMES Collaboration], Phys. Rev. D 71, 012003 (2005) [arXiv:hep-ex/0407032].

[59] D. Diakonov, V. Petrov, P. Pobylitsa, M. V. Polyakov and C. Weiss, Nucl. Phys. B 480 (1996) 341 [arXiv:hep-ph/9606314].

[60] B. Dressler, K. Goeke, M. V. Polyakov and C. Weiss, Eur. Phys. J. C 14 (2000) 147 [arXiv:hep-ph/9909541].

[61] B. Dressler, K. Goeke, M. V. Polyakov, P. Schweitzer, M. Strikman and C. Weiss, Eur. Phys. J. C 18, 719 (2001) [arXiv:hep-ph/9910464].

[62] A. V. Efremov, K. Goeke and P. V. Pobylitsa, Phys. Lett. B 488 (2000) 182 [arXiv:hep-ph/0004196].

[63] A. Bacchetta, R. Kundu, A. Metz and P. J. Mulders, Phys. Rev. D 65 (2002) 094021 [arXiv:hep-ph/0201091].

[64] M. Burkardt, Phys. Rev. D 69, 057501 (2004) [arXiv:hep-ph/0311013], Phys. Rev. D 69, 091501 (2004) [arXiv:hep$\mathrm{ph} / 0402014]$.

[65] D. Hasch [HERMES Collaboration], private communication.

[66] The COMPASS Collaboration, "Common Muon and Proton Apparatus for Structure and Spectroscopy", CERN/SPLC 96-14, SPSC/P 297.

[67] P. Lenisa and F. Rathmann et al. [PAX Collaboration], arXiv:hep-ex/0505054.

F. Rathmann et al., Phys. Rev. Lett. 94, 014801 (2005) [arXiv:physics/0410067], eConf C0409272, 014 (2004) [arXiv:hepex/0412063].

[68] M. Anselmino, V. Barone, A. Drago and N. N. Nikolaev, Phys. Lett. B 594, 97 (2004) [arXiv:hep-ph/0403114]. A. V. Efremov, K. Goeke and P. Schweitzer, Eur. Phys. J. C 35, 207 (2004) [arXiv:hep-ph/0403124], and arXiv:hep$\mathrm{ph} / 0412427$.

[69] Andy Miller, remark at "The International Workshop on Transverse Polarisation Phenomena in Hard Processes" (Transversity 2005), Villa Olmo (Como), 7-10th. September 2005.

[70] M. Anselmino et al., arXiv:hep-ph/0511017.

[71] J. C. Collins, A. V. Efremov, K. Goeke, M. Grosse Perdekamp, S. Menzel, B. Meredith, A. Metz and P. Schweitzer, preprint RUB-TPII-12-05, in preparation; and arXiv:hep-ph/0510342.

[72] Otherwise, for $p_{\text {Siv }}^{2} \rightarrow 0$ in (5) the Gaussian $\frac{\exp \left(-\mathbf{p}_{T}^{2} / p_{\text {Siv }}^{2}\right)}{\pi p_{\text {Siv }}^{2}} \rightarrow \delta^{(2)}\left(\mathbf{p}_{T}\right)$. Then $f_{1 T}^{\perp(1) a}(x) \rightarrow 0$ and the Sivers SSA (8) would vanish.

[73] For historical correctness we mention that previously (19) was discussed in the framework of (simple versions of) chiral models [47]. However, the way in which (19) was obtained there was shown to be incorrect [48]. Recently, in Ref. [49] a (more sophisticated version of a) chiral model with vector mesons obeying a hidden local flavour symmetry was discussed, in which the Sivers function obeys (19). 